\title{
Outcome of relapses of nephroblastoma in patients registered in the SIOP/GPOH trials and studies
}

\author{
HARALD REINHARD $^{1}$, ANDREA SCHMIDT ${ }^{2}$, RHOIKOS FURTWÄNGLER ${ }^{2}$, \\ IVO LEUSCHNER ${ }^{3}$, CHRISTIAN RÜBE ${ }^{4}$, DIETRICH VON SCHWEINITZ ${ }^{5}$, \\ ANDREAS ZOUBEK ${ }^{6}$, FELIX NIGGLI ${ }^{7}$ and NORBERT GRAF ${ }^{2}$
}

\author{
${ }^{1}$ Department of Pediatric Oncology, Asklepios Hospital Sankt Augustin; ${ }^{2}$ Department of Pediatric \\ Oncology, University Hospital Homburg/Saar; ${ }^{3}$ Department of Paidopathology, University Hospital Kiel; \\ ${ }^{4}$ Department of Radiotherapy, University Hospital Homburg/Saar; ${ }^{5}$ Department of Pediatric Surgery, \\ University Hospital Munich, Germany; ${ }^{6}$ Department of Pediatric Oncology, St. Anna Children's Hospital \\ Vienna, Austria; ${ }^{7}$ Department of Pediatric Oncology, Children's Hospital Zürich, Switzerland
}

Received January 29, 2008; Accepted April 7, 2008

DOI: 10.3892/or_00000029

\begin{abstract}
We registered 170 relapses in 1392 children with nephroblastoma in the SIOP/GPOH trials. The study aimed to evaluate prognostic factors for outcome in relapsed patients. Age, gender, initial stage, metastatic disease, local stage, histology, time to relapse and tumour volume were analysed for their prognostic relevance. Overall survival after relapse was $48 \%$ (median follow-up 5 years). Relapses were local in $28 \%$, metastatic in $57 \%$ and combined in $15 \%$. The median age of the cohort was 4.5 years whereas patients in complete continuous remission were significantly younger (3.1 years, $\mathrm{p}=0.001)$. Patients with initial stage I and II showed a significantly better prognosis than children with stage III ( 57 vs. $31 \%$, p=0.008). Patients with high-risk tumours had a much poorer prognosis than those with intermediate and lowrisk tumours ( 58 vs. $31 \%, \mathrm{p}=0.003$ ). Children with recurrence within 6 months after diagnosis had a poorer outcome than children relapsing later on (54 vs. $22 \%, \mathrm{p}=0.0001)$. The tumour volume initially and after preoperative chemotherapy did not have any influence on outcome. Patients with isolated distant metastasis had a significantly better outcome than those with local and combined relapses $(\mathrm{p}=0.001)$. In conclusion, factors for poor prognosis after relapse are early relapse, local stage III, high-risk histology and combined relapse.
\end{abstract}

Correspondence to: Dr Harald Reinhard, Asklepios Hospital, Pediatric Haematology and Oncology, D-53757 Sankt Augustin, Germany

E-mail: h.reinhard@asklepios.com

Key words: nephroblastoma, relapses, SIOP patients, risk groups, prognostic factors

\section{Introduction}

Wilms' tumour in childhood can be cured with current treatment approaches exceeding $85 \%$ event-free survival. In case of relapse, a subgroup of the patients has a poor prognosis of $<30 \%$. Since the first analysis of relapsed patients by the National Wilms Tumour Study Group (NWTS) (1), efforts have been made to improve the outcome of these patients. The introduction of drugs such as ifosfamide, carboplatinum and etoposide was promising (2), but did not ameliorate the outcome of certain subgroups. High-dose chemotherapy with autologous stem cell rescue is still under discussion for those relapsed patients with a poor outcome (3-5). A prospective randomised trial is still warranted. The pre-treatment regimen plays an important role in the case of relapse as patients initially treated with only two drugs can be cured to a significant extent (6). To evaluate the prognostic factors for relapsed patients diagnosed in Germany, Austria and partly Switzerland, we analysed all relapses that had occurred between 1989 and 2003 in patients treated according to the SIOP 9/GPO, SIOP 93-01/GPOH and SIOP 2001/GPOH (International Society of Pediatric Oncology, Gesellschaft für Pädiatrische Onkologie und Hämatologie) protocol.

\section{Materials and methods}

From 1989 until 2003, a total of 1392 patients with histologically proven Wilms' tumour were registered in the German SIOP/GPOH studies. A relapse occurred in 68 out of 375 SIOP 9 patients, 93 out of 705 SIOP 93-01/GPOH patients and in 9 out of 142 SIOP 2001/GPOH patients. The relapse rate was $12 \%$ for the three studies. The data of these 170 individuals, of whom 87 were male and 83 female, were recorded at the GPOH database. Initial staging was adapted to the recent SIOP staging system: patients with stage II $\mathrm{N}^{+}$ were classified as stage III retrospectively. Treatment was given according to the recommendations of the study center. All patients were treated with chemotherapy. Surgery was performed if possible to achieve a complete remission for 
Table I. Characteristics of the patients.

\begin{tabular}{|c|c|c|c|c|}
\hline & Relapse & $\%$ of relapse & Continuous remission (CR) & $\%$ of $\mathrm{CR}$ \\
\hline Total & 170 & 100.0 & 1222 & 100.0 \\
\hline \multicolumn{5}{|l|}{ Gender } \\
\hline Male & 87 & 51.0 & 598 & 48.0 \\
\hline Female & 83 & 49.0 & 624 & 52.0 \\
\hline \multicolumn{5}{|l|}{ Age (years) } \\
\hline Mean & $6.1 \pm 6.2$ & & $3.8 \pm 3.9$ & \\
\hline Median & 4.5 & & 3.1 & \\
\hline \multicolumn{5}{|l|}{ Tumor volume (ml) } \\
\hline Mean & $549 \pm 361$ & & $434 \pm 395$ & \\
\hline Median & 466 & & 350 & \\
\hline \multicolumn{5}{|l|}{ Clinical stage } \\
\hline I-III & 119 & 70.0 & 1063 & 87.0 \\
\hline IV & 51 & 30.0 & 159 & 13.0 \\
\hline \multicolumn{5}{|l|}{ Local stage } \\
\hline I-II & 106 & 62.0 & 990 & 81.0 \\
\hline III & 64 & 38.0 & 232 & 19.0 \\
\hline \multicolumn{5}{|l|}{ Histology } \\
\hline Low risk & 3 & 1.8 & 4 & 2.5 \\
\hline Completely necrotic & 3 & 1.8 & 4 & 2.5 \\
\hline Intermediate risk (type) & 95 & 56.0 & 973 & 79.7 \\
\hline Mixed & 45 & 26.7 & 432 & 35.4 \\
\hline Epithelial & 6 & 3.5 & 80 & 6.5 \\
\hline Stromal & 4 & 2.3 & 124 & 10.2 \\
\hline Regressive & 40 & 23.5 & 337 & 27.6 \\
\hline High risk & 72 & 42.2 & 217 & 17.8 \\
\hline Blastemal type & 36 & 21.1 & 166 & 13.6 \\
\hline Diffuse anaplasia & 36 & 21.1 & 51 & 4.2 \\
\hline
\end{tabular}

local and/or metastatic disease. Irradiation was only applied to the relapsed area if not given in the first-line treatment. Drugs that were used depended on the risk profile of the disease and the drugs used during the initial treatment. In high-risk patients carboplatin, etoposide and ifosfamide or cyclophosphamide were the main drugs used, whereas in low-risk patients actinomycin, vincristine and doxorubicin were still given. The cumulative dose of doxorubicin did not exceed $400 \mathrm{mg} / \mathrm{m}^{2}$. The median follow-up time of the cohort was 5 years. Blastemal type tumours after pre-operative chemotherapy were defined retrospectively as high-risk tumours, according to the revised SIOP classification (7).

In the first-line treatment, the patients had received doxorubicin in intermediate risk tumours of local stage II and III and in high-risk tumours of all stages within the three analysed studies. Radiotherapy was given to patients with stage III of intermediate risk and stage II and III of high-risk histology.

In our analysis, we initially compared the characteristics of the relapsed patients with those of children in continuous complete remission using descriptive statistical methods. We then looked for prognostic features in relapsed patients. The probabilities of overall survival (OS) were estimated by the Kaplan Meier method (8). Variables analysed were age, gender, initial clinical stage, metastatic disease, local stage, histology, time to relapse and tumour volume. Risk ratios were estimated using multivariate Cox models (9) with $95 \%$ confidence interval. For statistical analysis we used the SPSS (Chicago, IL) software, version 10.

\section{Results}

The median age of the 170 patients at initial diagnosis was 4.5 years and the mean age $6.1 \pm 6.2$. The patients were significantly older than those in complete continuous remission (CCR) (median 3.1, mean 3.8 \pm 3.9 years). Of the relapsed patients, 87 were male and 83 female. For 149 relapsed patients, the tumour volume at primary diagnosis and the volume reduction by initial treatment was available. The relapsed patients had a mean tumour volume at diagnosis of 


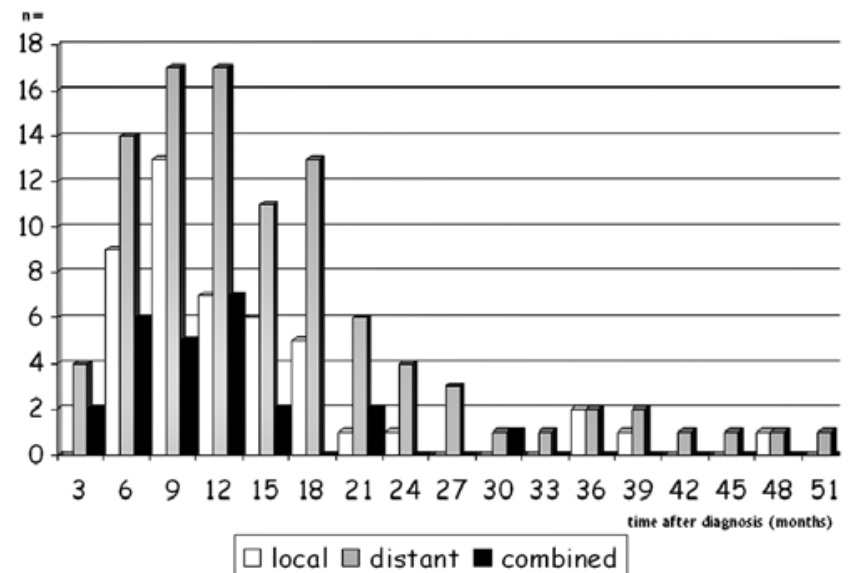

Figure 1. Frequency of relapses of the 170 patients, split for the type of relapse.

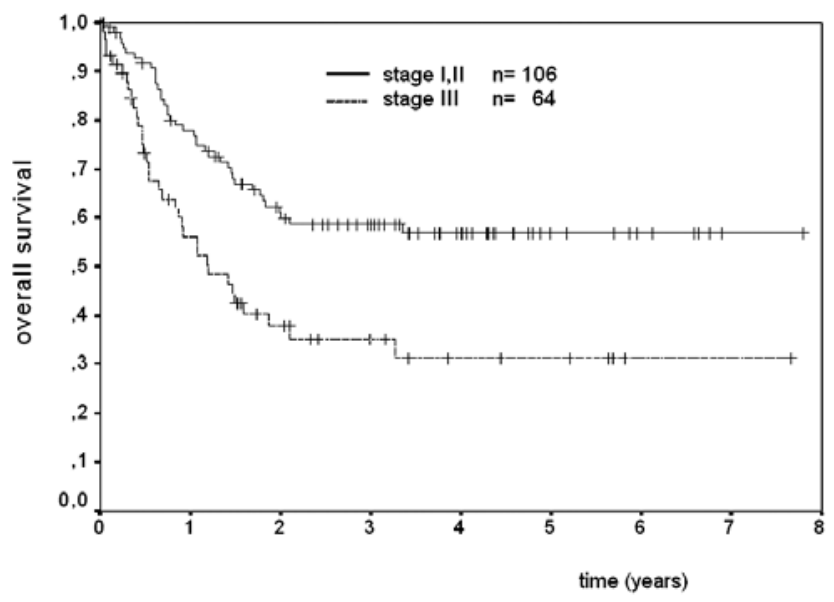

Figure 2. Outcome of the patients depends on the local stage. Patients with initial stage I and II have a significantly better prognosis $(\mathrm{OS}=57 \%)$ than those with stage III $(\mathrm{OS}=31 \%), \mathrm{p}=0.0008$.

$549 \pm 361 \mathrm{ml}$ and a median volume of $466 \mathrm{ml}$, whereas patients staying in CCR had a mean volume of $434 \pm 396 \mathrm{ml}$ and a median volume of $350 \mathrm{ml}(\mathrm{p}=0.001)$. After pre-operative chemotherapy, the tumour volume differed significantly between the two groups (mean $333 \pm 319$ vs. $206 \pm 255$ and median 247 vs. 126, $\mathrm{p}<0.001$ ). The clinical stage at the first diagnosis in relapsed patients was higher with $30 \%$ metastatic manifestations. Local stages showed a higher number of nonresectable tumours (stage III) in relapsed patients compared to children in CCR. The characteristics of the patients are demonstrated in Table I. High-risk tumours were more often diagnosed in relapsed patients including anaplastic and blastemal predominant tumours (Table I).

Most of the relapses were isolated metastases; $57 \%$ of the patients had distant metastasis, $15 \%$ a combined relapse and in $28 \%$, a local recurrence of the nephroblastoma was diagnosed. Patients with initial stage I had a local recurrence in $38 \%$, compared to $20 \%$ in patients with stage II and III. Of the 170 relapsed patients, 59\% had received doxorubicin in the first line treatment, while $42 \%$ of the patients in

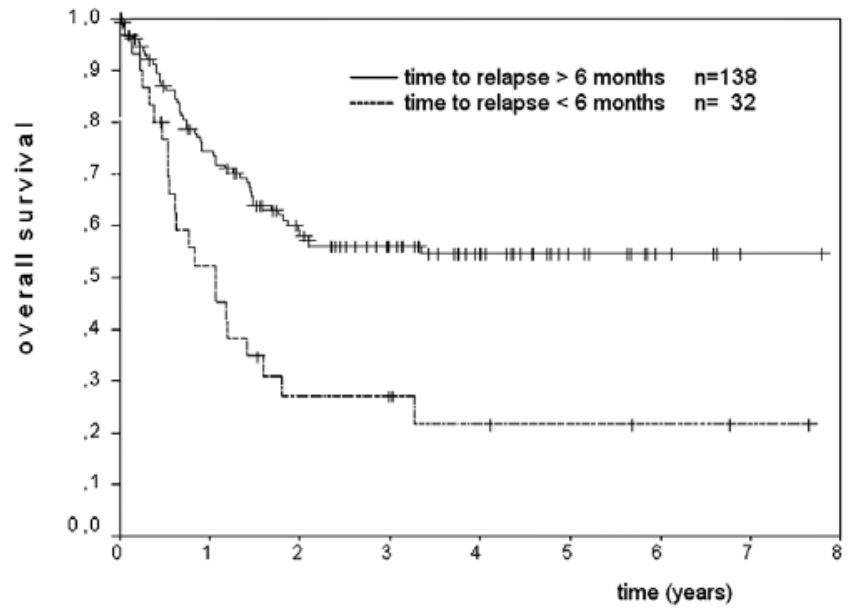

Figure 3. The time to relapse is a significant risk factor for relapsed patients (local and metastatic event). Overall survival is better in patients with recurrence $>6$ months after diagnosis (OS 54 vs. 22\%, p=0.0004).

continuous remission had been treated with anthracyclines. Patients with initial stage II (doxorubicin pre-treated, no radiotherapy) were represented equally in the two analysed groups (22\% of relapses and $23 \%$ of continous remissions). Radiotherapy was previously applied in $37 \%$ of the relapses and only in $19 \%$ of the patients in continuous remission.

The mean time to relapse was $1.1 \pm 0.8$ years and the median time to relapse was 0.9 years. In Fig. 1, the frequency of patients regarding time of relapse is shown divided by the type of relapse. The relapses $(>50 \%)$ occur within the first year after diagnosis.

The overall survival (OS) of the 170 children is $48 \%$ with a mean follow-up of 5 years. Prognosis is independent of gender, with males having an OS of $49 \%$ and females of $47 \%$. Regarding age, children $>4$ years showed a significantly better outcome with an OS of $57 \%$ compared to $42 \%$ for children $<4$ years. The initial clinical stage at diagnosis was also without prognostic relevance. Patients with metastatic disease at the time of initial diagnosis did not show a worse prognosis than childen with primary local disease (OS 52 vs. 44\%, p>1). However, the local stage at diagnosis is a relevant parameter for outcome after relapse. Patients with resectable tumours (stage I and II) had a significantly better prognosis than those with local stage III (OS 57 vs. 31\%, p=0.001) (Fig. 2). The histology of the tumour is also of prognostic relevance. Patients with a high-risk histology had a poorer outcome with an OS of $31 \%$ compared to those with standard risk tumours (OS 58\%, $\mathrm{p}=0.003$ ). Concerning the time to relapse, we found a significant difference of OS and EFS between early relapses $<6$ months from the time of diagnosis and relapses $>6$ months (Fig. 3). The type of relapse was another significant prognostic factor for outcome. Patients with isolated local or metastatic recurrence were doing better than those with a combined relapse (Fig. 4).

The multivariate analysis of the prognostic factors is summarized in Table II. Significant risk factors for outcome are local stage III, high-risk histology and combined relapses according to the multivariate Cox regression model. Although the time to relapse was a significant factor in the Kaplan-Meier 
Table II. Multivariate analysis of prognostic factors for 170 patients with relapsed Wilms' tumor.

\begin{tabular}{lcccc}
\hline Prognostic factor & Number & 5-year overall survival estimate (\%) & Relative risk (95\% CI) & P-value \\
\hline Age $(>4$ years) & 105 & 42 & $1.07(0.5-2.3)$ & 0.85 \\
Metastasis at relapse & 114 & 46 & $1.15(0.5-2.5)$ & 0.72 \\
Local stage III & 64 & 31 & $2.60(1.1-5.9)$ & 0.02 \\
Histology high-risk & 57 & 31 & $3.35(1.4-7.8)$ & 0.005 \\
Time to relapse $(<6$ months $)$ & 32 & 22 & $0.50(0.2-1.3)$ & 0.14 \\
Tumor volume $(>500 \mathrm{ml})$ & 70 & 38 & $1.37(0.4-4.2)$ & 0.58 \\
Combined relapse & 25 & 12 & $4.79(1.6-14.5)$ & 0.006 \\
\hline
\end{tabular}

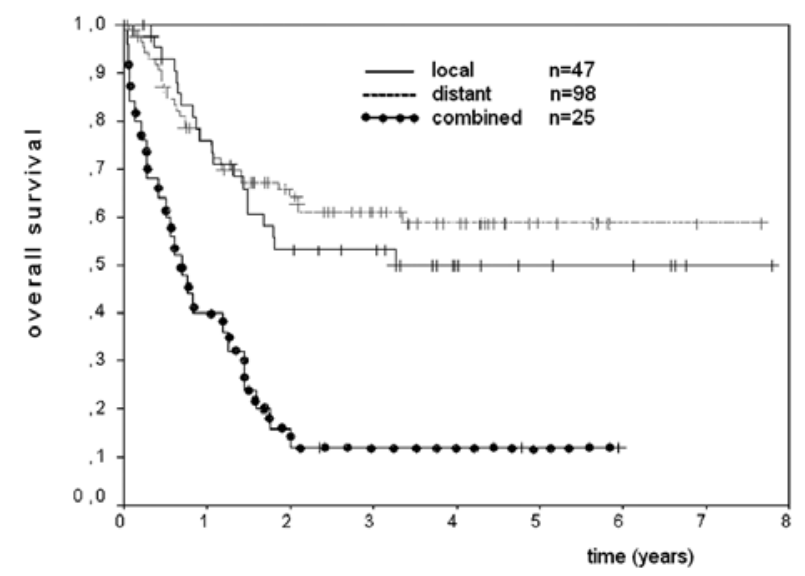

Figure 4. Patients with combined relapses $(\mathrm{OS}=12 \%)$ have a poorer prognosis than those with either isolated local $(\mathrm{OS}=50 \%)$ or distant relapses $(\mathrm{OS}=59 \%)$. The difference is significant $(\mathrm{p}<0.001)$.

analysis, it was not in the Cox regression with no elevation of the relative risk (RR).

\section{Discussion}

Patients with nephroblastoma have a realistic chance of being cured even in the situation of relapse. The results of our study confirm the observations of other groups (1). Since the 1980s, the outcome has improved gradually with the use of new effective drugs against nephroblastoma $(10,11)$. Patients pretreated with only two drugs have quite a good outcome after recurrence of the tumour since treatment (6). In previous studies, the characteristics that predispose for a relapse of the tumour have been defined, which we can confirm to a large extent. Of prognostic relevance are the age of the patient, clinical stage at diagnosis of the primary tumour, the local stage and histology. A new factor in our study is the tumour volume at diagnosis and after pre-operative chemotherapy, which was also shown to be significant. Other factors such as the presence of nephroblastomatosis in the tumour, influence of radiotherapy delay and surgery-related factors were found in literature (12-14), but were not part of our study.

The profile of the relapses in our study shows a predominance of metastatic events, either isolated or combined. Only a minority of the patients suffered from isolated local recurrence. This observation is in agreement with previous studies
$(10,13)$ and has been enhanced for NWTS patients without prior radiotherapy (6). Only 18 out of the 58 patients with initial stage I and II had a local recurrence, whereas the remainig 40 patients had metastatic or other events. Patients with stage I had more frequent local relapses in our study than patients with stage II and III, which may be due to local radiotherapy. However, patients with stage II that did not receive radiotherapy previously, had an identical local relapse rate $(20 \%)$ similar to patients with stage III who were previously irradiated. The efficacy and importance of doxorubicin can be documented in stage II patients without radiotherapy. We did not find a higher relapse rate in this group.

The overall survival in our study is $48 \%$ with a median follow-up of 5 years. The results are comparable with results from other groups. A Swedish series of 13 relapsed patients, that had been pre-treated with SIOP protocols (15) showed that 6 out of the 13 patients were long-term survivors after relapse. The results of St. Jude's Hospital are similar (16), with an overall survival of $63 \%$. In this study, the time period before 1984 including 34 relapsed patients is compared with the recent experience in 20 patients. The authors conclude that the new treatment strategies may improve the outcome of relapsed patients in NWTS patients. Their results show progress over the last two decades in contrast to the analysis of Grundy et al (1). This study gives an overall survival of $30 \%$ in 367 NWTS-2 and NWTS-3 patients. Certain subgroups had a better prognosis exceeding OS $40 \%$. The authors provide a very helpful analysis of prognostic factors after relapse. Similar results were published by the United Kingdom Children's Cancer Study Group (UKCCSG) for relapsed patients of the WT 1 Study (17). Of 71 relapses only 17 survived, and patients with an unfavorable histology had a very poor outcome of $<10 \%$.

In our study, we analysed a number of prognostic factors, which were regarded as relevant for outcome after tumour recurrence. In accordance with former studies, high local stage, high-risk histology, early event and combined site of relapse were found as significant prognostic parameters (18). The importance of the local stage and the previous treatment has recently been examined and published by NWTS (6). This study shows that patients being treated with only 2 drugs (vincristine and actinomycin D) have an excellent prognosis with an EFS of $71 \%$ and OS of $81 \%$, respectively. In our study population, the OS of patients with stage I and II was $57 \%$. This result, however, must take into acount that first-line SIOP treatment of stage II includes doxorubicin, and the use 
of this drug is limited in the situation of relapse for reasons of cardiac toxicity.

The relevance of the site of relapse is controversial. In our cohort, patients with isolated lung metastasis and isolated local recurrence had a significantly better prognosis than those with combined relapses. In the analysis of NWTS-2 and NWTS-3 patients (1), those with isolated lung metastasis had a better outcome than patients with local recurrence or other events. Patients with initial stage I and II showed no difference in outcome for local or distant relapses (11).

Patients with a high-risk histology have a high risk of suffering from a relapse and prognosis in this situation is very poor. The OS of this subgroup with an OS of $31 \%$ in our study is quite good compared to other studies. The NWTS patients reached a $10 \%$ OS and in the St. Jude's study, none of the nine patients with an unfavourable histology survived $(13,16)$. A promising treatment strategy for this subset of patients with a very poor prognosis has yet to be found.

Taking into account former studies as well as our own experiences, a group of patients can be defined as having multiple adverse prognostic factors in the case of relapse. These patients may benefit from more aggressive treatment regimens with new effective substances. Abu-Ghosh et al (19) reported a response rate to ICE (ifosfamide, carboplatin, etoposide) treatment of $82 \%$ in 11 patients with a high-risk relapse of nephroblastoma. The 3-year event-free survival of these patients was better than in former studies and reached $63.3 \%$, but the toxicity of the regimen was high. As these drugs are part of the first-line treatment in SIOP high-risk strategy, their use for this patient group may be less effective.

High-dose chemotherapy (HDC) with autologous stem cell rescue has been used in several trials for recurrent nephroblastoma $(4,5)$. The most recent study includes 13 patients showing a 4-year EFS of $60 \%$ and an OS of $73 \%$ (3). HDC can be effective in high-risk relapses of Wilms' tumour, but the question is still open as to whether this treatment is better than conventional chemotherapy. This question can only be answered by a randomized trial where patients will receive either conventional chemotherapy or HDC with stem cell rescue. This approach is initiated as a transatlantic cooperative study.

In conclusion, a protocol for relapses of nephroblastoma should stratify the patients according to their individual risk factor. Most important are local stage at diagnosis, histology, site of relapse and time to relapse. Besides surgical and radiotherapeutic approaches, depending on pre-treatment, patients with the highest risk profile should be included in a controlled and randomised trial for HDC.

\section{Ackowledgements}

We thank all participating centres in Germany, Austria and Switzerland, that have entered their patients into the GPOH trials.

\section{References}

1. Grundy P, Breslow N, Green DM, et al: Prognostic factors for children with recurrent Wilms' tumor: results from the second and third National Wilms' Tumor Study. J Clin Oncol 7: 638$647,1989$.

2. Miser JS and Tournade MF: The management of relapsed Wilms tumor. Hematol Oncol Clin North Am 9: 1287-1302, 1995.
3. Campbell AD, Cohn SL, Reynolds M, Seshadri R, Morgan E, Geissler G, Rademaker A, Marymount M, Kalapurakal J, Haut PR, Duerst R and Kletzel M: Treatment of relapsed Wilms' tumor with high-dose therapy and autologous hematopoietic stem-cell rescue: the experience at Children's Memorial Hospital. J Clin Oncol 22: 2885-2890, 2004.

4. Pein F, Michon J, Valteau-Couanet D, Quintana E, Frappaz D, Vannier JP, Philip T, Bergeron C, Baranzelli MC, Thyss A, Stephan JL, Boutard P, Gentet JC, Zucker JM, Tournade MF and Hartmann O: High-dose melphalan, etoposide, and carboplatin followed by autologous stem-cell rescue in pediatric high-risk recurrent Wilms' tumor: a French Society of Pediatric Oncology study. J Clin Oncol 16: 3295-3301, 1998.

5. Kremens B, Gruhn B, Klingebiel T, Hasan C, Laws HJ, Koscielniak E, Hero B, Selle B, Niemeyer C, Finckenstein FG, Schulz A, Wawer A, Zintl F and Graf N: High-dose chemotherapy with autologous stem cell rescue in children with nephroblastoma. Bone Marrow Transplant 30: 893-898, 2002

6. Green DM, Cotton CA, Malogolowkin M, Breslow NE, Perlman E, Miser J, Ritchey ML, Thomas PR, Grundy PE, D'Angio GJ, Beckwith JB, Shamberger RC, Haase GM, Donaldson M, Weetman R, Coppes MJ, Shearer P, Coccia P, Kletzel M, Macklis R, Tomlinson G, Huff V, Newbury R and Weeks D: Treatment of Wilms tumor relapsing after initial treatment with vincristine and actinomycin D: a report from the National Wilms Tumor Study Group. Pediatr Blood Cancer 48: 493-499, 2007.

7. Vujanic GM, Sandstedt B, Harms D, Kelsey A, Leuschner I and de Kraker J: SIOP Nephroblastoma Scientific Committee: Revised International Society of Paediatric Oncology (SIOP) working classification of renal tumors of childhood. Med Pediatr Oncol 38: 79-82, 2002.

8. Kaplan EL and Meier P: Non-parametric estimation from incomplete observations. J Am Stat Assoc 54: 457-481, 1958.

9. Cox DR: Regression models and life tables (with discussion). JR Stat Soc 34: 187-220, 1972.

10. Pein F, Tournade MF, Zucker JM, Brunat-Mentigny M, Deville A, Boutard P, Dusol F, Gentet JC, Legall E, Mechinaud F, et al: Etoposide and carboplatin: a highly effective combination in relapsed or refractory Wilms' tumor-a phase II study by the French Society of Pediatric Oncology. J Clin Oncol 12: 931-936, 1994.

11. Marina MN, Wiliams JA, Meyer WH, et al: Refining therapeutic strategies for patients with resistant Wilms' tumor. Am J Ped Hematol Oncol 16: 296-300, 1994.

12. Bergeron C, Iliescu C, Thiesse P, Bouvier R, Dijoud F, Ranchere-Vince D, Basset T, Chappuis JP, Buclon M, Frappaz D, Brunat-Mentigny $\mathrm{M}$ and Philip T: Does nephroblastomatosis influence the natural history and relapse rate in Wilms' tumour? A single centre experience over 11 years. Eur J Cancer 37: 385-391, 2001.

13. Kalapurakal JA, Li SM, Breslow NE, Beckwith JB, Macklis R, Thomas PR, D'Angio GJ, Kim T, de Lorimier A, Kelalis P, Shochat S, Ritchey M, Haase G, Hrabovsky E, Otherson HB, Grundy P and Green DM: National Wilms' Tumor Study Group: Influence of radiation therapy delay on abdominal tumor recurrence in patients with favorable histology Wilms' tumor treated on NWTS-3 and NWTS-4: a report from the National Wilms' Tumor Study Group. Int J Radiat Oncol Biol Phys 57: 495-499, 2003.

14. Shamberger RC, Guthrie KA, Ritchey ML, et al: Surgeryrelated factors and local recurrence of Wilms Tumor in National Wilms Tumor Study 4. Ann Surg 229: 292-297, 1999.

15. Kullendorf C-M: Relapses in Wilms tumour. Pediatr Surg Int 19: 635-638, 2003.

16. Dome J, Liu T, Krasin M, et al: Improved survival for patients with recurrent Wilms tumor: The experience at St. Jude Children's Research Hospital. J Ped Hematol Oncol 24: 192$198,2002$.

17. Groot-Loonen JJ, Pinkerton CR, Morris-Jones PH and Pritchard J: How curable is relapsed Wilms' tumour? The United Kingdom Children's Cancer Study Group. Arch Dis Child 65: 968-970, 1990.

18. Pein F, Rey A, de Kraker J, Ludwig R, Pötter R and Godzinsky J: Multivariate Analysis of Adverse Prognostic Factors (APF) in Children with recurrent Wilms' tumor (WT) after initial treatment according to SIOP-6 or SIOP-9 strategies. Med Ped Oncol 33: 170, 1999 .

19. Abu-Ghosh AM, Krailo MD, Goldman SC, Slack RS, Davenport V, Morris E, Laver JH, Reaman GH and Cairo MS: Ifosfamide, carboplatin and etoposide in children with poor-risk relapsed Wilms' tumor: a Children's Cancer Group report. Ann Oncol 13: 460-469, 2002. 